\title{
Fortalecimiento y mejora continua de la Revista Médica Hondureña
}

\author{
Continuous improvement and strengthening of the Revista Médica Hondureña
}

El proceso de renovación de la Revista Médica Hondureña ha sido constante durante varios años. Sin embargo, en 2019 se ha dado un impulso al fortalecimiento y mejora en todo el proceso editorial, con énfasis en los parámetros bibliométricos, uso de estándares de publicación y el proceso de revisión por pares. Además, se comenzó a trabajar en el desarrollo de un sitio web dedicado que facilite el acceso a recursos de información a los autores, revisores y lectores (http://revistamedicahondurena.hn/). Todas estas acciones están enmarcadas en la misión y visión de la Revista que les comparto a continuación. Misión: Difundir y comunicar información científica inédita fundamentados en principios éticos y de calidad aplicables a la práctica clínica, la salud pública e investigación para la salud, para fomentar y apoyar la investigación científica y la educación médica continua, especialmente del gremio médico nacional. Visión: Ser una revista que difunda conocimiento científico inédito con alta calidad, prestigio e integridad científica, accesible a nivel nacional e internacional y con amplia representatividad en el campo de la práctica clínica, la salud pública e investigación para la salud.

En relación a los parámetros bibliométricos, se ha priorizado la puntualidad de la Revista y la estructura de su contenido, aspirando a que al menos $40 \%$ corresponda a los trabajos científicos originales. Este aspecto en sí mismo es un reto ya que la publicación es la culminación de un ciclo de investigación en el cual se debe contar con un sistema institucional y nacional de investigación para la salud fortalecido, con gobernanza sólida, con marco legal y regulatorio inclusivo, que responda a principios y valores éticos, con priorización de la investigación innovadora de acuerdo a las necesidades de la población y acceso a recursos financieros que promuevan el desarrollo continuo científico y tecnológico. La falta de investigaciones éticas y de calidad limitan directamente la disponibilidad de artículos originales y de manera indirecta la disponibilidad de todos los otros tipos de artículos que publica la Revista. En los últimos años, aunque se editaron solamente dos números regulares, denominados números 1-2 y 3-4, se trató de incluir el contenido correspondiente a cuatro números. Actualmente, la publicación continuará siendo trimestral, cuatro números en un año. Se ha impulsado la utilización de estándares de publicación tales como las recomendaciones para la conducta, informe, edición y publicación de trabajos académicos (http://www.icmje.org/recommendations/), las guías de la Red EQUATOR (www.equator.org), las directrices para la integridad ética y moral de las publicaciones del comité de ética en la publicación (https://publicationethics.org/), el registro de protocolos, ensayos clínicos y de los investigadores y la declaración de conflictos de interés. En el proceso de revisión por pares, el Consejo Editorial se ha fortalecido al contar con editores asociados nacionales e internacionales con una amplia gama de experiencia clínica, salud pública, epidemiología e investigación, así como con asistentes editoriales capacitados.

Todos estos aspectos se irán abordando de manera escalonada, conforme a una ruta estratégica acordada con la Secretaría de Asuntos Educativos y Culturales del Colegio Médico de Honduras. El apoyo de entes internacionales como el Centro Latinoamericano y del Caribe en Ciencias de la Salud (BIREME) ha contribuido a la mejora de la Revista a través de la capacitación sobre Buenas Prácticas en el Proceso Editorial de Revistas Científicas (2019). Los principales retos planteados se irán mitigando con el apoyo de todos los aliados estratégicos, incluyendo los autores, lectores, revisores, la Biblioteca Médica Nacional, los organismos internacionales y la Junta Directiva y la magna Asamblea del Colegio Médico de Honduras. En 2020, la Revista Médica Hondureña cumple 90 años y la visualizamos como una publicación científica fortalecida, líder en su área.

\author{
Jackeline Alger, MD, PhD \\ Directora \\ Revista Médica Hondureña
}

Dirección para correspondencia: Dra. Jackeline Alger, MD, PhD

Correo electrónico: jackelinealger@gmail.com 